\title{
Signature of exotic particles in light by light scattering
}

\author{
G. Tavares-Velasco ${ }^{1}$ \\ Departamento de Física, CINVESTAV, Apartado Postal 14-740, 07000, México \\ D. F., México \\ J.J. Toscano ${ }^{2}$ \\ Facultad de Ciencias Físico Matemáticas, Benemérita Universidad Autónoma de \\ Puebla, Apartado Postal 1152, Puebla, Pue., México.
}

\begin{abstract}
We discuss the implications on light by light scattering of two kind of exotic particles: doubly charged scalar bosons and doubly charged fermions; the virtual effects of a nonstandard singly charged gauge boson are also examined. These particles, if their masses lie in the range $0.1-1.0 \mathrm{TeV}$, will have a clear signature in the future linear colliders. The present analysis has the advantage that it depends only on electromagnetic symmetry, so it is applicable to any model which predicts this class of particles. In particular, our results have interesting consequences on left-right models and their supersymmetric extension.
\end{abstract}

The major dream in particle physics is a final theory of elementary interactions. The standard model (SM) leaves many unaddressed questions, so it is only one step toward the achievement of such a theory. In the attempt to take one step forward many extensions have been conjectured, resulting in the prediction of new particles, whose experimental evidence would point in the right direction. In this letter we will examine the implications of different kinds of exotic particles on light by light scattering [1]-[3], which has been proposed recently as an useful mode to detect virtual effects of new physics at the future linear colliders (LC) [4]. Our study includes doubly charged scalar bosons and fermions, as well as a singly charged gauge boson heavier than the SM one. The mass range studied will be $0.1-1 \mathrm{TeV}$, which would be at the reach of LC [5]. Since the $\gamma \gamma$ scattering amplitude is proportional to the electric charge

1 E-mail: gtv@fis.cinvestav.mx

2 E-mail: jtoscano@fcfm.buap.mx

Preprint submitted to Elsevier Preprint 11 July 2017 
factor $Q^{4}$, the contribution of particles with charge greater than the unity, in terms of the positron charge, would enhance dramatically the respective cross section, resulting in a distinctive signal of new physics. Moreover, the structure of the cross section is entirely dictated by the spin of the particles circulating in the loop and by electromagnetic symmetry, thus it is not necessary to make further assumptions about a specific model. As a consequence, our analysis is applicable to any model which predicts such particles. Nevertheless, the main motivation of our work resides in two popular extensions of the standard model, namely left-right symmetric models (LRM) [6] and their supersymmetric extension (SUSYLR) [7], where these exotic particles are a natural prediction and they might provide the most distinctive signature of these models. We will begin by presenting a brief outline of these extensions.

Doubly charged Higgs bosons emerge in many extensions of the SM Higgs sector [8]. Unacceptable effects on the electroweak tree level relation $\rho \equiv$ $m_{W}^{2} /\left(\cos \theta_{W} m_{Z}^{2}\right) \approx 1$ may arise from exotic representations which have triplets or higher representations with a neutral member, but it is possible to overcome this problem by recoursing to extra assumptions. This is the case in a popular class of models with both doublets and triplets, where a custodial SU(2) symmetry is invoked to protect the $\rho$ relation [9]. In general, the $\rho$ problem is avoided in representations without neutral members or either in models where the vacuum expectation value (VEV) of the neutral component of the Higgs multiplets vanishes. More complicated possibilities arise in extensions of the $\mathrm{SU}(2) \times \mathrm{U}(1)$ gauge group. For instance, the minimal version of LRM, which are based on the $\mathrm{SU}(2)_{\mathrm{L}} \times \mathrm{SU}(2)_{\mathrm{R}} \times \mathrm{U}(1)_{\mathrm{B}-\mathrm{L}}$ gauge group, requires the presence of one bidoublet as well as left and right triplets. These models predict the existence of new physics at an intermediate scale provided by the VEV of the neutral component of the right triplet, parity-symmetry would be restored at this scale. The VEV's of the neutral components of the bidoublet are identified with the Fermi scale. As far as the left triplet is concerned, it is only required to preserve left-right symmetry, and its neutral component can get a VEV constrained to be small to maintain the $\rho$ relation. In its minimal realization, LRM predict fourteen physical Higgs bosons but we are only interested on the doubly charged bosons as it is likely that they give a clear signature of new physics. In the gauge sector, the only new contribution to light by light scattering comes from a charged right-handed gauge boson. The low-energy implications of LRM depend strongly on the structure and the vacuum stability of the Higgs potential. It was shown that a careful analysis of the most general Higgs potential consistent with the low-energy data might not lead to new physics at a low scale [10]. However, if new physics featured by discrete symmetries is imposed, the most delicate terms of the Higgs potential can be eliminated, allowing the existence of a scale that would be accessible at LC. In particular, in these scenarios some Higgs bosons might be light, with masses of the order of the Fermi scale. 
With regard to doubly charged fermions, they appear first in natural lepton models [11]. More recently, they have emerged naturally from the supersymmetric extension of LRM. At this respect, there is the belief that supersymmetry (SUSY), and in particular its minimal low-energy realization, the supersymmetric standard model (MSSM), is a natural candidate to supersede the SM. Although MSSM offers solution to problems not explained by the SM it has many undesirable features, namely it predicts large $C P$ violating effects, it allows the presence of baryon and lepton number violating terms in the lagrangian, and it forbids the existence of massive neutrinos if global $R$-parity is to be conserved. These problems may be cured by considering the supersymmetric extension of LRM, SUSYLR [12], which has also the attractiveness of the presence of a low-energy scale $m \sim M_{R}^{2} / M_{\text {Planck }}$, where $M_{R}$ is the scale of left-right symmetry breaking. It happens that some singly and doubly charged Higgs scalars and the respective superpartners have their masses proportional to $m$. Very recently it has been argued that two interesting possibilities arise depending if the vacuum of the theory does or does not conserve $R$-parity [13]. When the vacuum conserves $R$-parity, low energy data set a lower limit on $M_{R}$ of about $10^{10} \mathrm{GeV}$. On the other hand, in the scenario where the vacuum state breaks $R$-parity spontaneously, it exists an upper limit of about $10 \mathrm{TeV}$ for $M_{R}$. It follows that even in the case of $M_{R}$ in the range $10^{10}-10^{12} \mathrm{GeV}$, there is the possibility of some light doubly charged Higgs bosons and Higgsinos. This is an important motivation to study the implications of doubly charged scalars and fermions at LC through light by light scattering.

The main virtue of light by light scattering is that to a certain extent it is a model-independent process: the structure of its amplitude is entirely dictated by the spin of the virtual particles as well as electromagnetic symmetry, and of course by the number of such particles. Therefore, the only dependence on a specific model is given by the mass and electric charge of its particle content. As was pointed out in [4], in the SM the helicity amplitudes of the $\gamma \gamma$ scattering are almost purely imaginary at high energies, precisely in the range where there is the possibility of observing the appearance of fields associated with models beyond the SM. There follows that if the contribution arising from additional charged particles has an appreciable imaginary part, the virtual effects would become evident through the interference with the SM contribution even if the respective contribution is too small to be detected by itself. Another interesting feature of light by light scattering is that the amplitude arising from loops with the same particle circulating on them turns out to be proportional to the charge factor $Q^{4}$, the consequence is that the cross section gets enhanced dramatically when the contribution of a doubly charged particle is considered. With all these properties, the process $\gamma \gamma \rightarrow \gamma \gamma$ provides an excellent mechanism to search for virtual effects of new physics at LC: the signature of a certain particle with a particular spin and charge depends only on its mass, and it is not necessary to consider model-dependent parameters or make further assumptions as it does the case when direct production is 
studied.

We will proceed to discuss our results. We have considered the implications on light by light scattering of doubly charged scalars and fermions with masses in the range $0.1-0.5 \mathrm{TeV}$. As far as the singly charged gauge boson is concerned, we have studied the case in which its mass is greater that the existing bound of $550 \mathrm{GeV}$, obtained if it is assumed a light right-handed neutrino [14]. For the purpose of this work, it is sufficient to analyze unpolarized cross sections. A more detailed study will be presented elsewhere [15], including polarized cross sections and the implications of the technical details of LC, together with the study of exotic particles not discussed in here: doubly charged gauge bosons and exotic quarks with charges $5 / 3 e$ and $-4 / 3 e$, which are predicted by some $\mathrm{SU}(3)_{\mathrm{c}} \times \mathrm{SU}(3)_{\mathrm{L}} \times \mathrm{U}(1)_{\mathrm{N}}$ models [16]. The helicity amplitudes of contributions of loops with scalars, fermions and gauge bosons are well known [2]-[4]. To obtain the cross section we have worked with the exact amplitudes without making any simplification but to consider the observable cross section, the integration has been constrained to $|\cos \theta| \leq 30^{\circ}$. The numerical analysis was done with the program FF [17].

The case where singly charged fermions and scalar bosons are involved was studied with details in [4], in the context of SUSY models $\square$. The remarkably properties of the $\gamma \gamma$ scattering acquire new dimensions when particles with a charge greater than the unity, in units of the positron charge, are involved. This is shown through Figs. 1-6, where we have plotted separately the contributions of each exotic particle for different values of its mass. In Fig. 1, it is shown the respective contribution as well as the interference between a doubly charged fermion and the SM contribution, scaled by the SM unpolarized cross section $\sigma_{\mathrm{SM}}$. It must be noticed that, though the virtual effects arise predominantly from the interference term, in the case of a light fermion with a mass of about $100 \mathrm{GeV}$ even its own contribution plays an important role. This seems to contradict the well known result that at energies about $300 \mathrm{GeV}$ the top contribution is negligible with respect to the $W_{L}$ term. The explanation resides in the powerful charge factor: as the helicity amplitudes for the contribution of a certain particle are proportional to the factor $Q^{4}$, the cross section arising from a doubly charged fermion turns out to be improved by the factor $2^{8} /\left(3(2 / 3)^{4}\right)^{2}=3^{6}$ in comparison with the case of an up-type quark, whereas the interference term gets enhanced by the factor $3^{3}$. When the mass $M_{\hat{\delta}_{++}}$of the doubly charged fermion is greater than $200 \mathrm{GeV}$, its contribution to the unpolarized cross section tends to be suppressed with respect to the interference term. It is also interesting to note that the virtual effects are always observed above the threshold $\sqrt{s} \geq 2 M_{\hat{\delta}_{++}}$. This fact is explained because of the character predominantly imaginary of the SM helicity ampli-

3 We do not show these results, but it must be noticed that we find a nice agreement with [4]. 
tudes at energies above $300 \mathrm{GeV}$, at the same time the new contributions are purely real below the threshold and complex above it. As the interference is given by $2 \Re\left(A_{\mathrm{SM}} A_{\mathrm{New}}\right)$, it follows that the virtual effects will become evident above the threshold. To realize the magnitude of the deviation from the SM, we have plotted in Fig. 2 the effect that would be observed if in addition to the SM particle content, a doubly charged fermion is included. It is clear that the signature of such an exotic particle would be very distinctive. The sensitivity of the unpolarized cross section at a linear collider running at energies in the range of $350-800 \mathrm{GeV}$ has been examined for the case of a singly charged fermion [4], it was found that for a chargino with a mass of $100-250 \mathrm{GeV}$ the signal varies between $3 \mathrm{SD}-1 \mathrm{SD}$. It is evident that in the case of a doubly charged fermion we should expect an important increment [15]. Although the signature of a doubly charged scalar is not as spectacular as that of a fermion with the same charge, the situation is also promising as it is depicted in Figs 3-4. In Fig. 3 it can be seen that the virtual effects come predominantly from the interference with the SM particles, the result is reflected on the deviation from the unpolarized cross section $\sigma_{\mathrm{SM}}$ (Fig. 4). If we compare this particular case with that of a singly charged scalar with about the same mass, we find that, since the dominant effect comes from interference, the deviation for a doubly charged scalar is larger for a factor of $2^{4}$. As a result, the possibility of observing the virtual effects of a relatively heavy doubly charged scalar would be increased as compared to the case of the singly charged scalar. Finally, for completeness we have studied the potential effects of a relatively light singly charged gauge boson. The motivation is that it has been noticed that the existence of such a particle would have important implications to elucidate some aspects of SUSYLR [13]. We have plotted in Figs 5-6 the deviation from the SM cross section as caused by a singly charged gauge boson with a mass in the range $0.55-1 \mathrm{TeV}$. It can be seen that, as expected, the enhancement of the cross section is not as important as the ones arising from doubly charged particles. However, at energies of about $2 \mathrm{TeV}$, the signal would be more important than the one coming from a doubly charged scalar. In contrast to the situation of fermions and scalars, where the deviation from the SM cross section is most important near the threshold, the one coming from a gauge boson is larger far beyond.

Our results show that the signature of exotic particles would be distinctive enough in $\gamma \gamma$ scattering to provide evidence of new physics. In particular, in the context of SUSYLR, an interesting implication is that a doubly charged Higgs scalar boson or a doubly charged Higgsino with masses in the range 100-500 GeV would produce a clear signature. This signal would be more distinctive at LC that the one which could provide a chargino or a sfermion with the same or even with a lighter mass. In addition, the existence of several doubly charged particles will enhance spectacularly the cross section and as a result particle counting might be possible through light by light scattering. If SUSYLR is realized in nature, then it would exist the possibility that low- 
energy remnant doubly charged Higgs bosons and doubly charged Higgsinos would exist, as it has been suggested recently in conjecturing some scenarios [13]. If this possibility became true, it is likely that this kind of particles would be discovered by direct production [18] by the time that LC would be a reality. In this situation, $\gamma \gamma$ scattering might be an effective process to probe the theory with a high precision.

In conclusion, due to its outstanding properties, light by light scattering rises as an invaluable process to search indirectly signals of doubly charged particles at the planned linear colliders. The most remarkably feature is that the signal arising from a certain particle depends only on its mass. As an alternative to direct production, where model-dependent parameters have to be considered, light by light scattering might offer the possibility of testing to a great detail the properties of new charged particles. In particular, it would aid also to elucidate some characteristics of a specific model, for instance the number of particles of a certain kind.

\section{Acknowledgements}

We acknowledge support from CONACYT and SNI (México).

\section{References}

[1] H. Euler and B. Kockel, Naturwissenchaften 23 (1935) 246; W. Heinsenberg and H. Euler, Z. Phys. 98 (1936) 714; P.A.M. Dirac, Proc. Cambridge Philos. Soc. 30 (1934) 150; W. Heinsenberg, Z. Phys. 90 (1934) 209; N. Kemmer and V.F. Weisskopf, Nature 137 (1936) 659.

[2] R. Karplus and M. Neuman, Phys. Rev. 80 (1950) 380; 83 (1951) 776; B de Tollis, Nuovo Cimento 35 (1965) 1182; V. Constantini, B. de Tollis and G. Pistoni, Nuovo Cimento A 2 (1971) 733.

[3] H. Fanchiotti, H.O. Girotti, and A. Sirlin, Lett. Nuovo Cimento A 2 (1971) 733; F. Boudjema, Phys. Lett. B 187 (1987) 362; X.-D. Jiang and X.-Zhou, Phys. Rev. D 47 (1993) 214; G. Jikia and A. Tkabladze, Phys. Lett. B 323 (1994) 453.

[4] G.J. Gounaris, P.I. Porfyriadis and F.M. Renard, Phys. Lett. B 452 (1999) 76; Eur. Phys. J. C 9 (1999) 673.

[5] I. Ginzburg, G.L. Kotkin, V.G. Serbo, and V.I. Telnov , Nucl. Instrum. and Methods 205 (1983) 47; I. Ginzburg et al., ibid. 219 (1984) 5 ; V. Telnov, ibid. A 294 (1990) 72; 355 (1995) 3; S. Kuhlman et al. hep-ex/9605011; D.A. Bauer, Int. J. Mod. Phys. A 11 (1996) 1637. 
[6] J.C. Pati and A. Salam, Phys. Rev. D 10 (1974) 275; R.N. Mohapatra and J.C. Pati, Phys. Rev. D 11 (1975) 566; 2558; G. Senjanovic and R.N. Mohapatra, Phys. Rev. D 12 (1975) 1502.

[7] M. Cvetic and J.C. Pati, Phys. Lett. B 135 (1984) 57; Y. Ahn, Phys. Lett. B 149 (1984) 337; R.M. Francis, M. Frank, and C.S. Kalman, Phys. Rev. D 43 (1991) 2369; R. Kuchimanchi and R.N. Mohapatra, Phys. Rev. D 48 (1993) 4352.

[8] J. Gunion, H. Haber, G. Kane, and S. Dawson, The Higgs Hunter's Guide, Addison Wesley, Reading. Ma. 1990.

[9] H. Georgi and M. Machacek, Nucl. Phys. B 262 (1985) 463; S. Chivukula and H. Georgi, Phys. Lett. B 182 (1986) 181; J.F. Gunion, R. Vega, and J. Wudka, Phys. Rev. D 42 (1990) 1673; 43 (1991) 2322.

[10] J.F. Gunion, J. Grifols, A. Mendez, B. Kayser, and F. Olness, Phys. Rev. D 40 (1989) 1546. N.G. Deshoande, J.F. Gunion, B. Kayser, and F. Olness, ibid. 44 (1991) 837.

[11] F. Wilczek and A. Zee, Phys. Rev. Lett. 38 (1977) 531.

[12] R.N. Mohapatra, Phys. Rev. D 34 (1986) 3457; A. Font, L. Ibanez, and F. Quevedo, Phys. Lett. B 228 (1989) 79; S. Martin, Phys. Rev. D 46 (1992) 2769. R.N. Mohapatra and A. Rasin, Phys. Rev. Lett. 76 (1996) 3490; R. Kuchimanchi, Phys. Rev. Lett. 76 (1996) 3486; R.N. Mohapatra, A. Rasin, and G. Senjanovic, Phys. Rev. Lett. 79 (1997) 4744. R.N. Mohapatra and G. Senjanovic, Phys. Rev. Lett. 44 (1980) 912.

[13] Z. Chako and R.N. Mohapatra, Phys. Rev. D 58 (1998) 015003; B. Dutta and R. N. Mohapatra, Phys. Rev. D 59 (1998) 015018; B. Dutta, R.N. Mohapatra, and D.J. Muller, Phys. Rev. D 60 (1999) 095005; C.S. Aulakh, A. Melfo, A. Rašin, and G. Senjanović, Phys. Rev. D 58 (1998) 115007; C.S. Aulakh, A. Melfo, and G. Senjanović, Phys. Rev. D 57 (1998) 4174.

[14] C. Caso et al., Review of Particle Physics, Eur. Phys. J. C3 (1999) 1.

[15] G. Tavares-Velasco and J. J. Toscano, in preparation.

[16] F. Pisano and V. Pleitez, Phys. Rev. D 46 (1992) 410; R. Foot, O.F. Hernandez, F. Pisano, and V. Pleitez, Phys. Rev. D 47 (1993) 4158.

[17] G. J. van Oldenborgh, Comp. Phys. Commun. 66 (1990) 1.

[18] F. Cuypers and S. Davidson, Eur. Phys. J. C 2 (1998) 503; G. Barenboim, K. Huitu, J. Maalampi, and M. Raidal, Phys. Lett. B 394 (1997) 132; K. Huitu, J. Maalampi, A. Pietilä, and M. Raidal, Nuc. Phys. B 487 (1997) 27; S. Chakrabarti, D. Choudhury, R.M. Godbole, and B. Mukhopadhyaya, Phys. Lett. B 434 (1998), 347. K. Huitu, J. Maalampi, and M. Raidal, Nuc. Phys. B 420 (1994) 449, M. Raidal, and P.M. Zerwas, Eur. Phys. J. C 8 (1999) 479. 

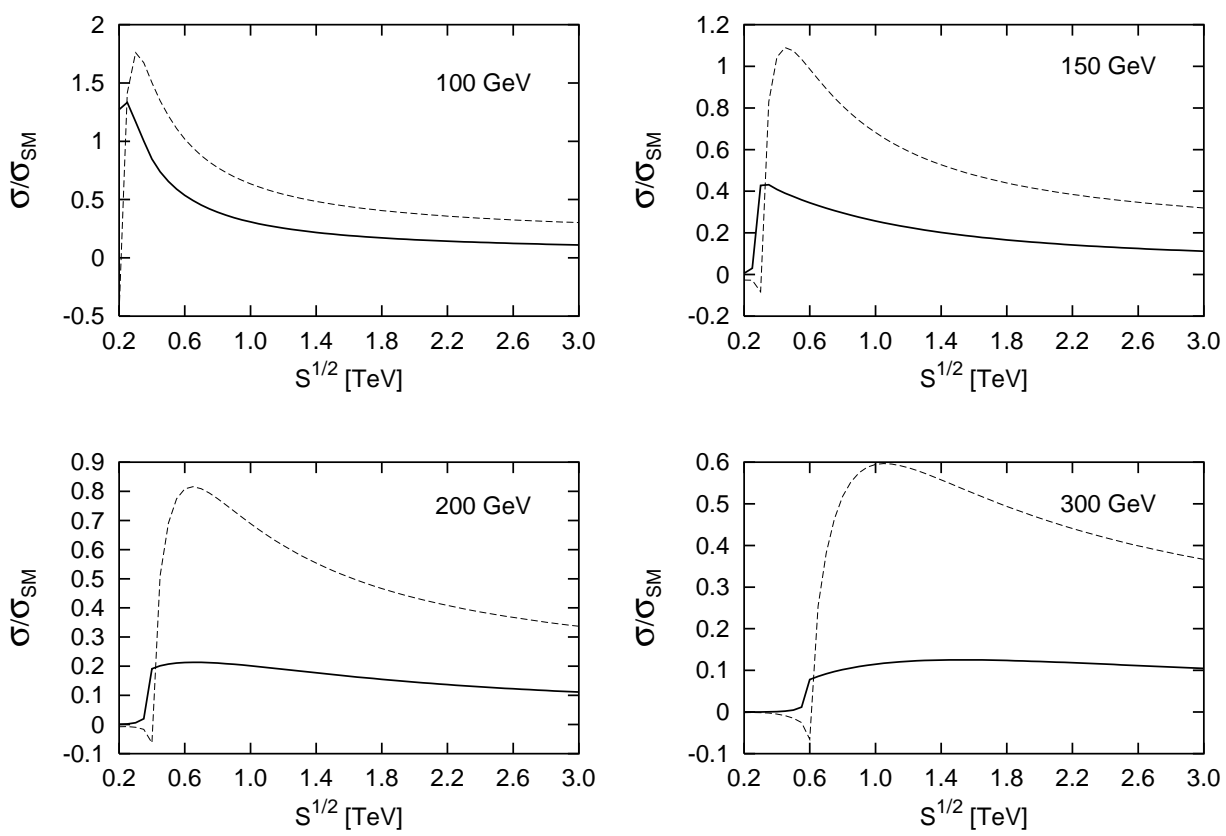

Fig. 1. Interference (dashes) between the SM amplitude and that of a doubly charged fermion $\hat{\delta}^{++}$as well as its contribution (solid line) to the unpolarized cross section, for different values of the fermion mass. The values are scaled by the SM unpolarized cross section.

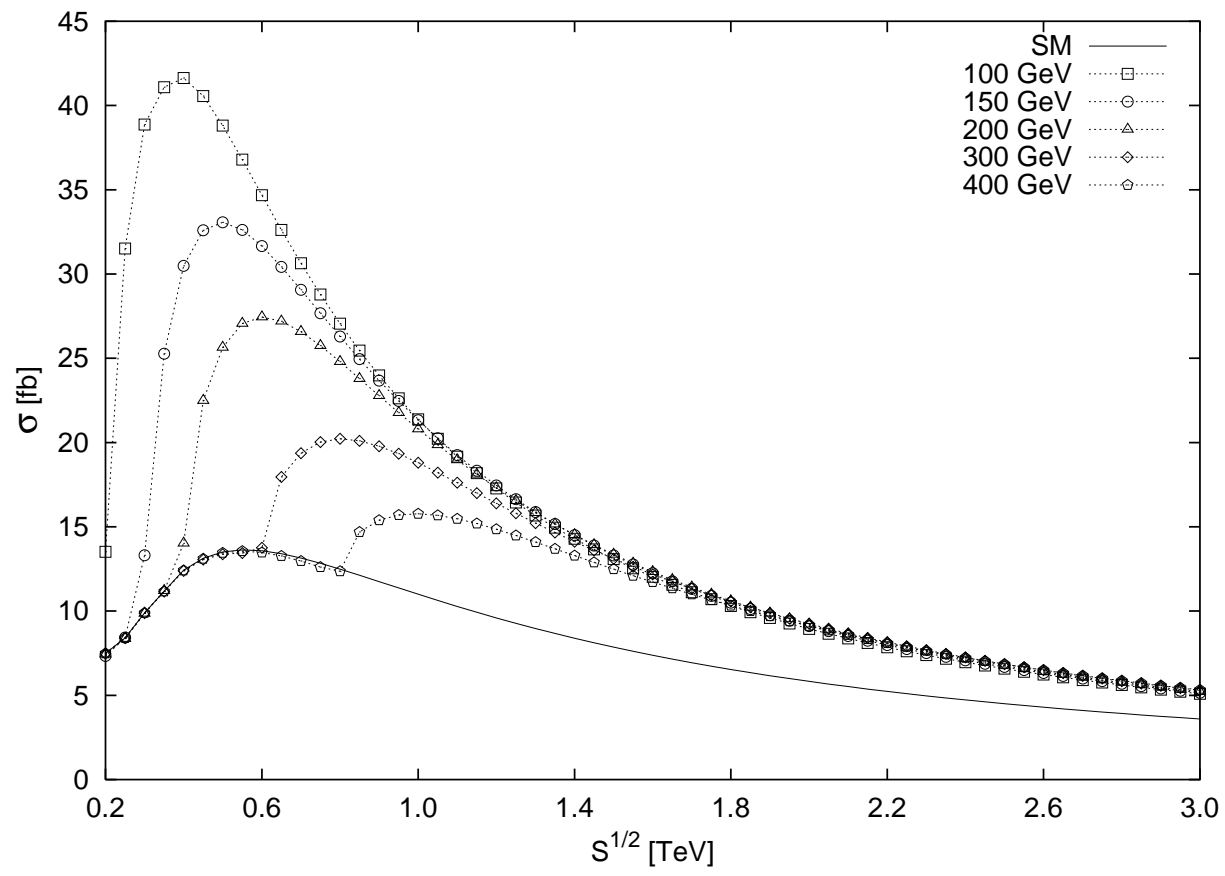

Fig. 2. Deviaton from the SM unpolarized cross section when a doubly charged fermion $\hat{\delta}^{++}$contributes, for different values of the fermion mass. 

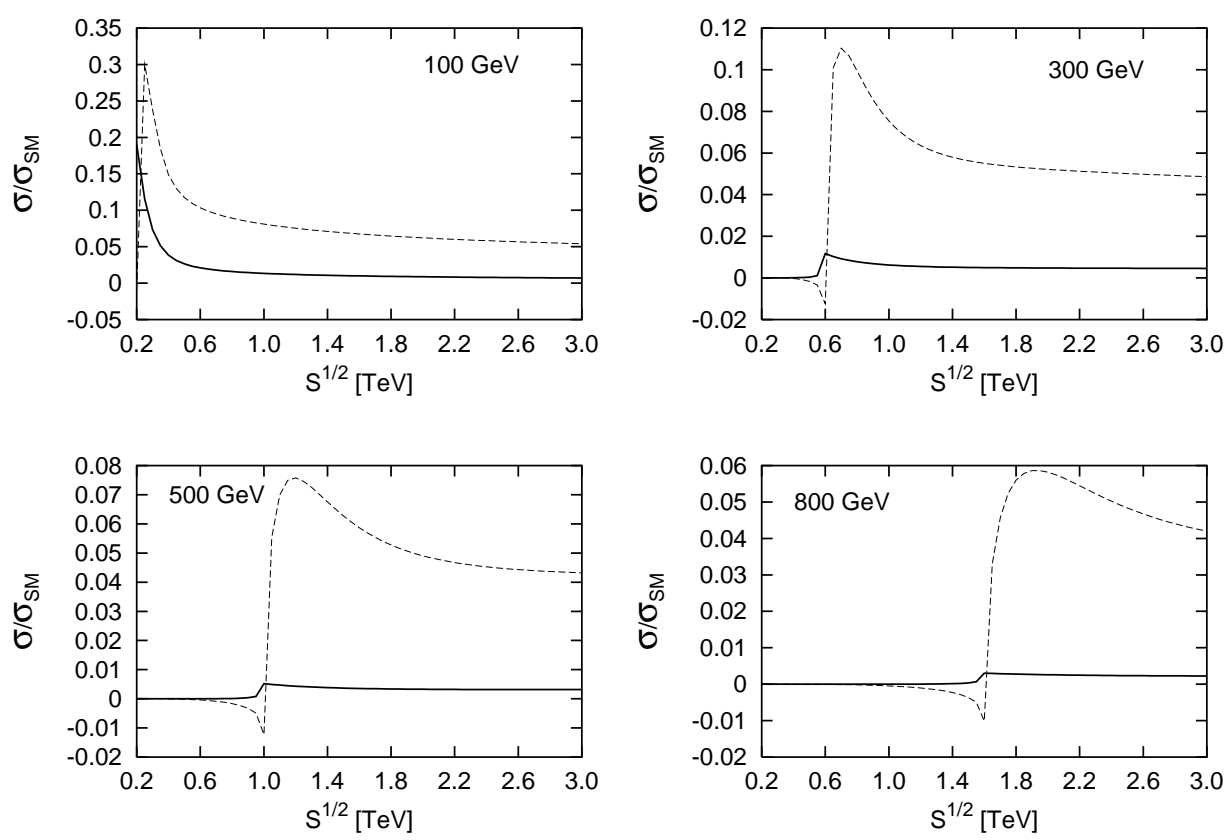

Fig. 3. The same as in Fig. 1 when a doubly charged scalar $\delta^{++}$is involved.

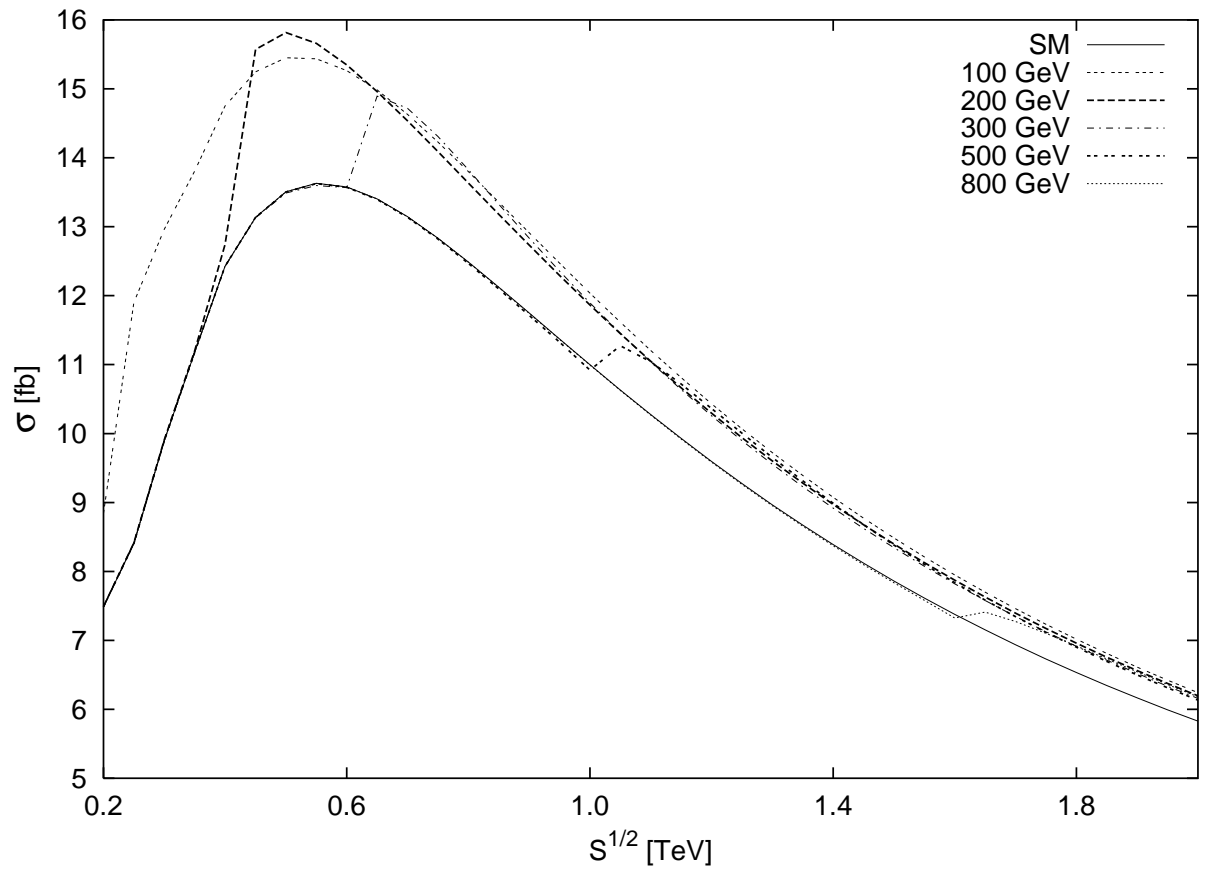

Fig. 4. The same as in Fig. 2 when a doubly charged scalar $\delta^{++}$is involved. 

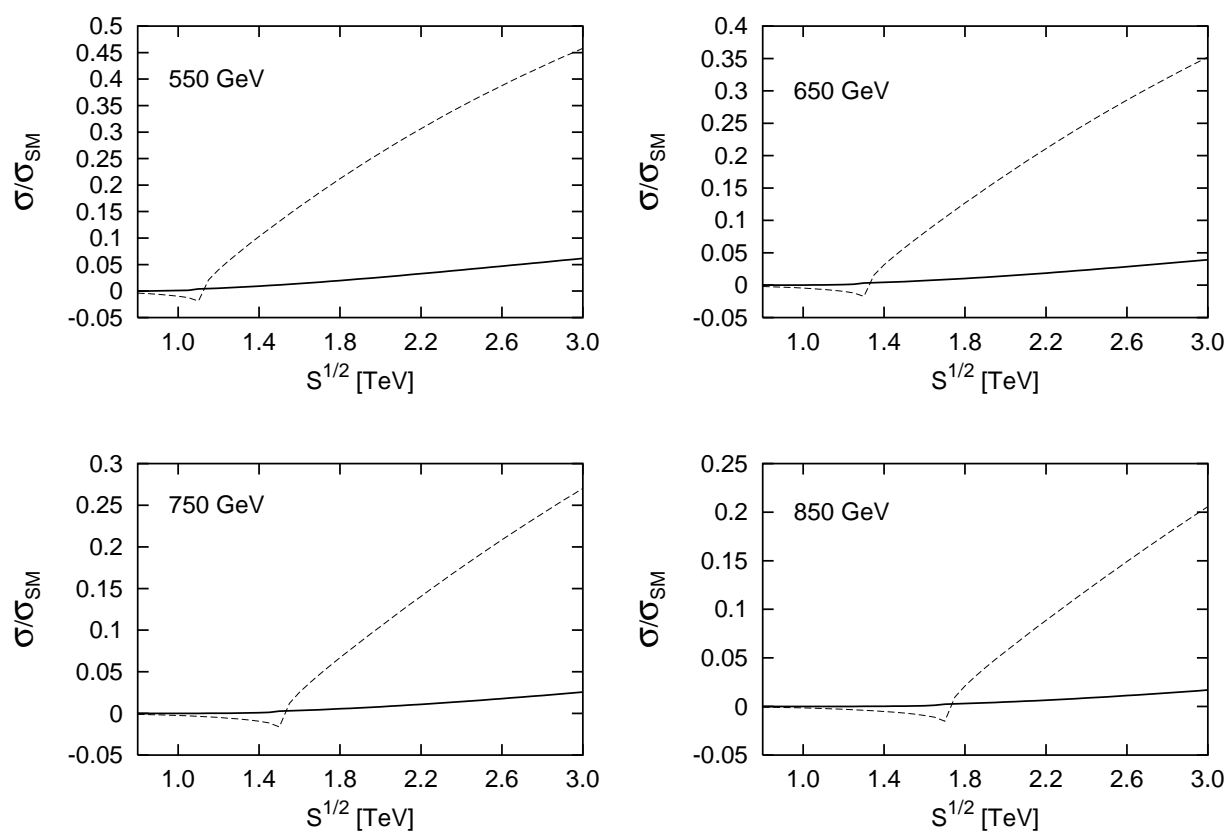

Fig. 5. The respective plot as in Fig. 1 when a singly charged gauge boson $W_{R}$ is involved.

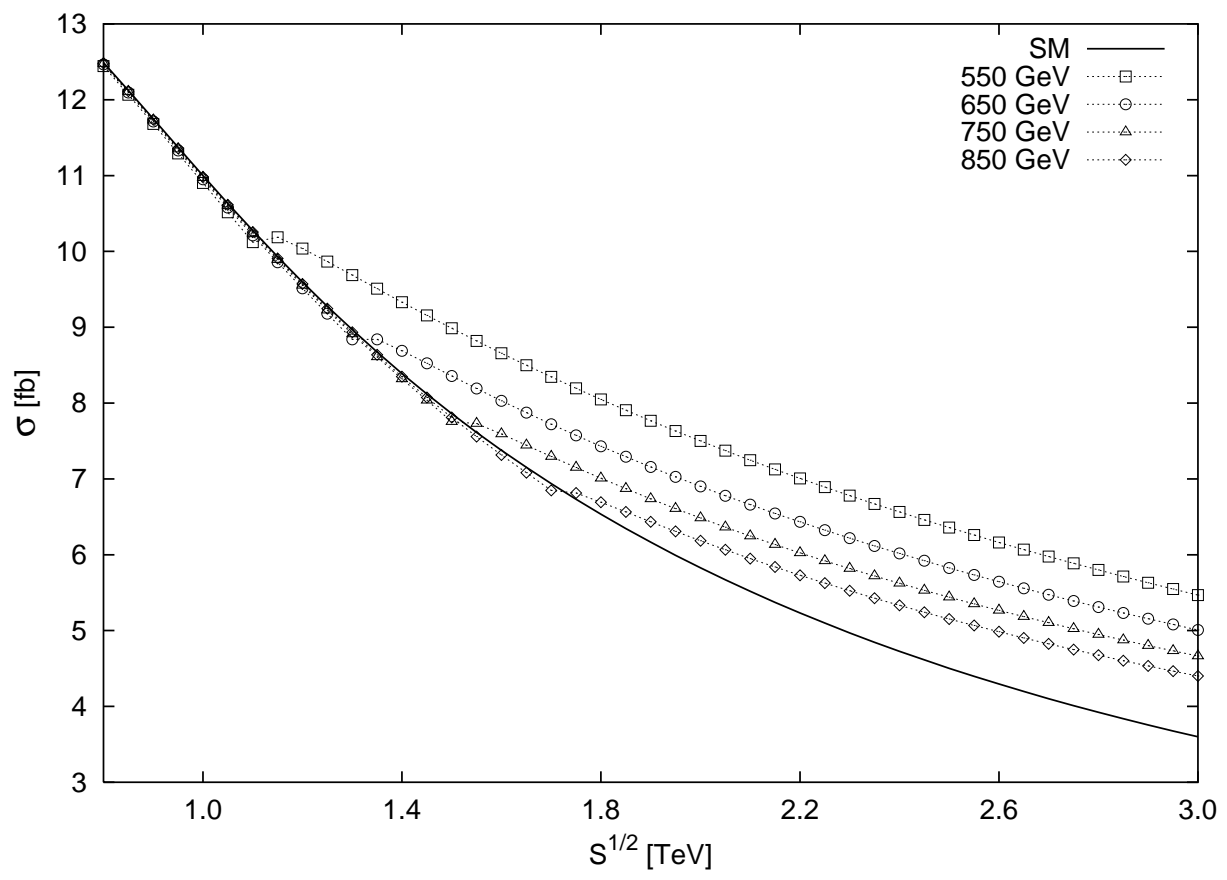

Fig. 6. The same as in Fig. 2 when a singly charged gauge boson $W_{R}$ is involved. 